\title{
A case of biphasic pulmonary blastoma presenting in a 55-year-old man
}

\author{
J onathan Pararajasingham ${ }^{1}$, David Lawrence ${ }^{1}$, Davide Patrini ${ }^{1}$, Mohamed A Gulamhussein ${ }^{1}$, \\ Elaine Borg ${ }^{2}$, Nikolaos Panagiotopoulos ${ }^{1}$ \\ 1. Department of Cardiothoracic Surgery, University College London Hospitals (UCLH), London, UK. 2. Department of \\ Histopathology, University College London Hospitals (UCLH), London, UK. \\ Correspondence: Dr. Jonathan Pararajasingham. Address: Department of Cardiothoracic Surgery, The Heart Hospital, \\ University College London Hospitals (UCLH), 16-18 Westmoreland Street, London W1G 8PH, UK. E-mail: \\ jpararaja@doctors.org.uk
}

Received: February 17, 2015

Accepted: March 26, 2015

Online Published: March 31, 2015

DOI : $10.5430 /$ crcp.v2n3p44

URL: http://dx.doi.org/10.5430/crcp.v2n3p44

\section{Abstract}

Pulmonary blastomas are rare malignant neoplasms, typically occurring in childhood. When diagnosed in adults, pulmonary blastoma is seen in the fourth decade of life, with a strong preponderance in female patients. We report an unusual case of a 55-year-old male patient diagnosed with a classic biphasic pulmonary blastoma.

\section{Key words}

Classic biphasic pulmonary blastoma, Rare lung tumours, Pulmonary malignancy

\section{I ntroduction}

Pulmonary blastoma is a rare form of lung cancer, typically diagnosed in children. When occurring in adults, pulmonary blastoma is seen in the fourth decade of life (mean age 43 years), with a strong preponderance in female patients ${ }^{[1]}$. We report an unusual case of a 55-year-old man who presented with exertional dyspnoea and was found to have an irregularly defined right middle lobe mass in his medical work-up. This mass was surgically resected and subsequently found to be a classic biphasic pulmonary blastoma.

\section{Case presentation}

A 55-year-old Caucasian man presented to the tuberculosis clinic with shortness of breath after walking a quarter of a mile, though was able to get up and down a flight of stairs. He denied chest pain, weight loss or recent travel. His past medical history was significant for non-active Hepatitis C and previous Hepatitis B infection. He was not diabetic, mildly asthmatic and smoked 3 "roll up" cigarettes a day. There was no relevant family history, and he was normocholesterolaemic and a treated hypertensive. On examination there were reduced breath sounds, more markedly over the right hemithorax.

A full blood count, renal and liver function, coagulation screen and an electrocardiogram were all unremarkable. On pulmonary function testing he had an FEV1 of 2.6 and TLCO of 74\%. A chest radiograph demonstrated an opacity in the 
right middle lobe suspicious of a mass lesion. A subsequent computed tomography (CT) chest confirmed a $3.5 \mathrm{~cm} \times$ $3.1 \mathrm{~cm}$ solid, irregular heterogeneous mass within the right middle lobe laterally (see Figure 1), with a further satellite nodule also within the middle lobe laterally measuring $0.5 \mathrm{~cm}$. 18F-fluorodeoxyglucose positron emission tomography/ computed tomography (FDG-PET/CT) found the main lesion to be FDG avid (see Figure 2). The adjacent peripheral 0.6 cm area of nodularity was not FDG-avid and therefore likely to be inflammatory rather than a satellite nodule. There was a mildly avid $0.9 \mathrm{~cm}$ hilar lymph node, but no evidence of distant disease.

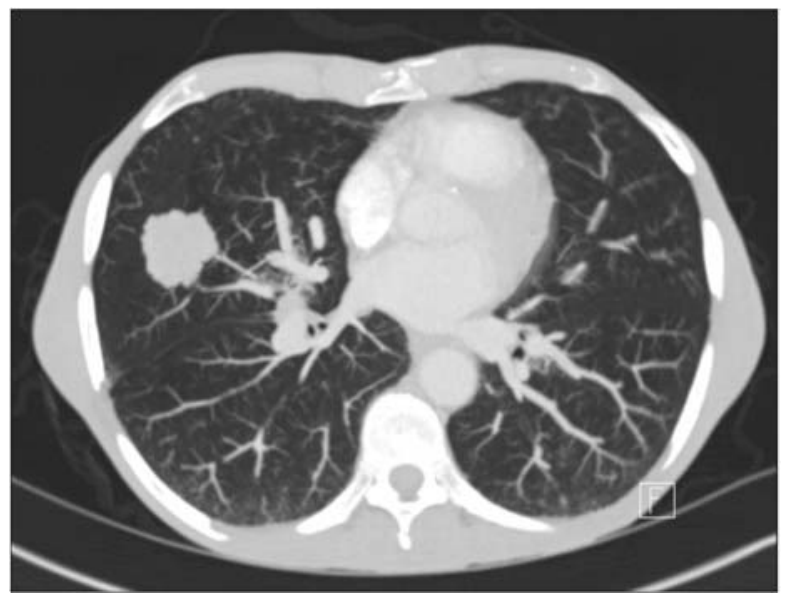

Figure 1. Computed tomography (CT) scan of the chest demonstrating the $3.5 \mathrm{~cm}$ mass within the right middle lobe

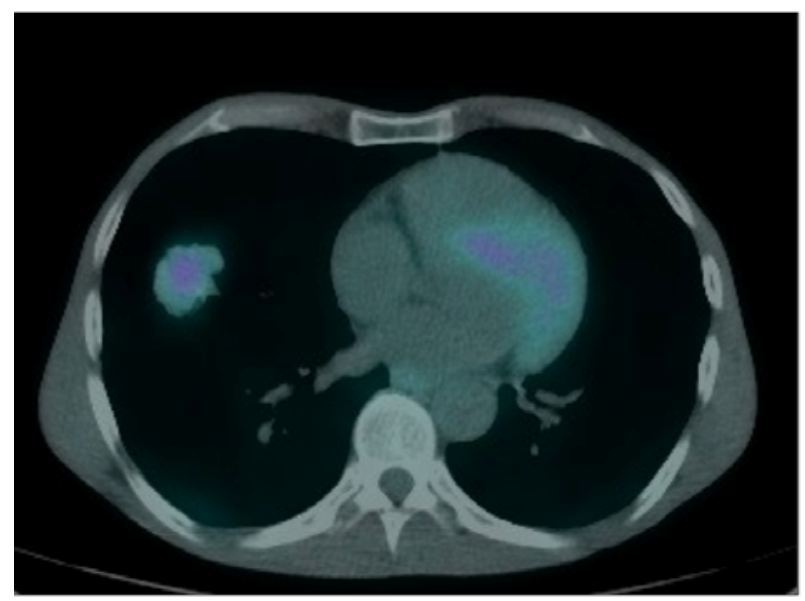

Figure 2. 18F-fluorodeoxyglucose positron emission tomography/computed tomography (FDG-PET/CT) demonstrating FDG uptake within the mass

He underwent a right-sided video-assisted thoracoscopic surgery (VATS) and middle lobectomy with an uneventful recovery. Histological analysis revealed an irregularly defined solid trimmed coloured tumour measuring $28 \mathrm{~mm} \times 20 \mathrm{~mm}$ $\times 35 \mathrm{~mm}$. No other tumours were found in the rest of the lobe. It was a biphasic tumour composed of atypical cells arranged in trabeculae, solid nests, glands, cords and papillary pattern (see Figure 3). The glandular cells were tall columnar with clear cytoplasm and subnuclear and supranuclear vacuoles at places. These tubular glands appeared to be surrounded by stroma and morules of cells with abundant eosinophilic cytoplasm. There was no evidence of lymphovascular space invasion.

Figure 3. Photomicrograph showing the microscopic appearance of a section of pulmonary blastoma. Note the gland formation and trabeculae lined by columnar cells, features that are typical for a diagnosis of pulmonary blastoma. The cytology of the tumour shows a high cell nuclear: cytoplasmic ratio, prominent nucleoli and glycogen-rich supra-nuclear vacuoles, which were PAS-positive and PASD-negative (not shown). Haematoxylin \& eosin (H\&E) ( $\times 40$ objective).

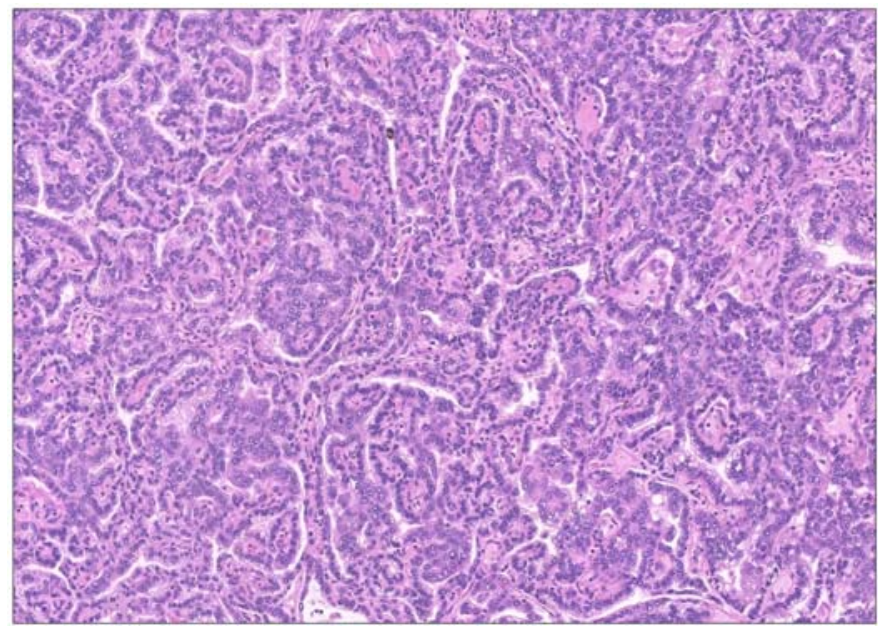

The epithelial tumour cells were positive for TTF1 and CK7 (see Figure 4). Vimentin was patchily and focally positive in the stromal component (see Figure 5). There was no evidence of infiltration of the visceral pleura (PL0). The bronchial and vascular resection margins were free of the tumour. The tumour was $10 \mathrm{~mm}$ from the bronchial resection margin and 
there was no evidence of metaplasia, dysplasia or invasive malignancy at the margin. The background lung showed emphysematous change. These findings were diagnostic of classic biphasic pulmonary blastoma.

Figure 4. Photomicrograph of a section of pulmonary blastoma, stained using immunohistochemistry (brown) with an antibody that localises to epithelial cytokeratin (CK7). Note the brown cytoplasmic staining of some of the cells of the tumour, highlighting its biphasic (mixed epithelial and mesenchymal) components ( $\times 10$ objective).

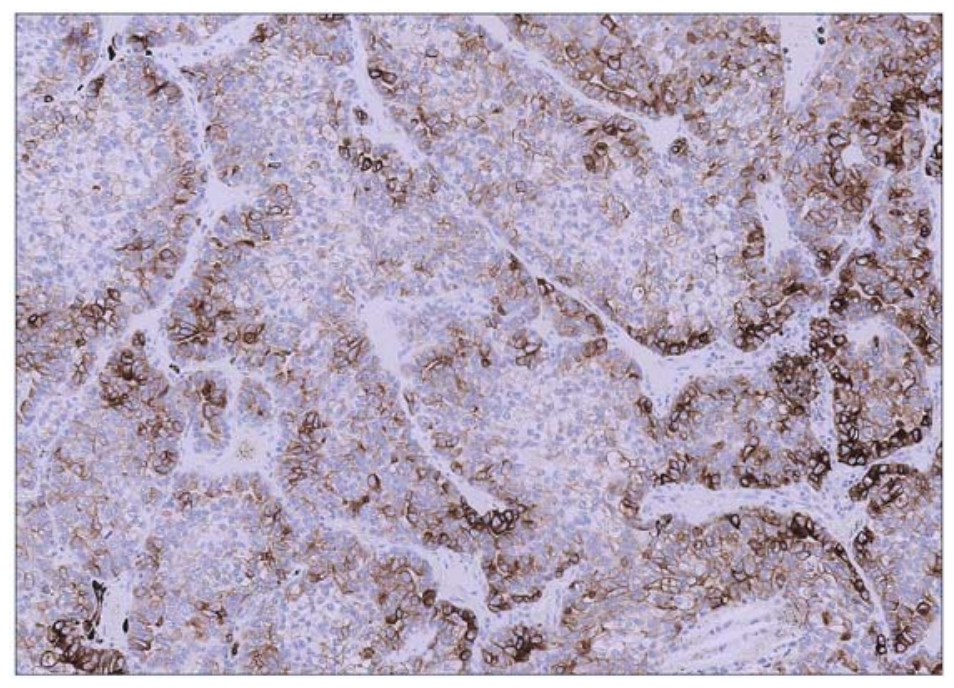

Figure 5. Photomicrograph of a section of pulmonary blastoma, stained using immunohistochemistry (brown) with an antibody that localizes to the mesenchymal cell marker, vimentin. Note the brown cytoplasmic staining of some of the cells of the tumour, highlighting its biphasic (mixed epithelial and mesenchymal) components $(\times 10$ objective $)$.

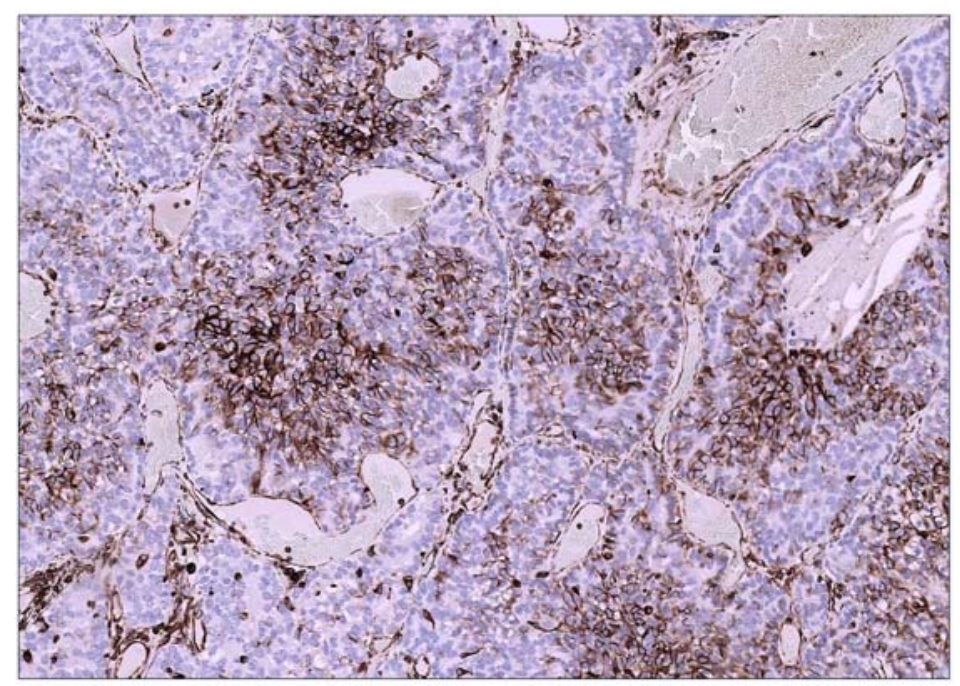

\section{Discussion}

Pulmonary blastomas are rare malignant neoplasms comprising $0.25 \%-0.5 \%$ of all primary lung neoplasms ${ }^{[2]}$. They are divided into three subcategories: classic biphasic pulmonary blastoma (CBPB), well-differentiated fetal adenocarcinoma (WDFA), and pleuropulmonary blastoma (PPB). PPB is currently regarded as a separate entity, referred to as the pulmonary blastoma of childhood ${ }^{[3]}$, whereas CBPB and WDFA typically occur in adults with a mean age of 43 years ${ }^{[4]}$. Since 1999 the WHO classification separates CBPB from the other two types, where it is now part spectrum of sarcomatoid carcinomas ${ }^{[5]}$.

Forty percent of pulmonary blastoma cases are asymptomatic. Typical symptoms include cough, haemoptysis, dyspnoea or chest pain, as a result of the tumour impinging on the bronchi or pleura. Clinical examination may reveal reduced breath sounds. Smoking is identified as a causative agent with over $80 \%$ of cases having a history of smoking. Chest radiography typically reveals a large, solitary, well-circumscribed, unilateral mass. Bronchoscopic biopsy is achievable in only $25 \%$ of cases due to the peripheral location of these tumours. 
Histologically, WDFA contains both malignant glands and benign appearing mesenchymal tissue and PPB contains malignant glands of embryonal appearance and benign appearing epithelium. CBPB however contains glands and mesenchymal tissue that are both embryonal and malignant ${ }^{[6]}$.

Surgical excision is the mainstay of treatment, though recurrence following resection is high. The efficacy of adjuvant chemotherapy and radiotherapy is not yet established, though there is some suggestion from the literature that when aggressive treatment includes postoperative chemotherapy and radiotherapy following surgery, it could be useful to prolong patient survival ${ }^{[4]}$.

The prognosis of pulmonary blastoma is very poor, with an overall five-year survival of $16 \%$. WDFA appears to have a better prognosis. Adverse prognostic factors include tumour size, biphasic type, recurrence, metastasis at initial presentation and lymph node metastasis.

Although extremely rare, pulmonary blastoma should be considered as part of the differential diagnosis of lung masses, with consideration being extended to older, male patients. As it is difficult to achieve a diagnosis based on radiological findings or bronchoscopic biopsy, early surgical excision is recommended, to establish histological diagnosis, prevent further disease progression and increase survival.

\section{Conflict of interests}

The authors declare no conflict of interests.

\section{References}

[1] Robert J, Pache JC, Seium Y, et al. Pulmonary blastoma: report of five cases and identification of clinical features suggestive of the disease. European Journal of Cardio-thoracic Surgery. 2002; 22(5): 708-711. http://dx.doi.org/10.1016/S1010-7940(02)00529-8

[2] Mistry, et al. Primary pulmonary blastoma of monophasic variety- diagnosis and management. Journal of Cardiothoracic Surgery. 2013; 8: 144. PMid: 23758909. http://dx.doi.org/10.1186/1749-8090-8-144

[3] Manivel JC, Priest JR, Watterson J, et al. Pleuropulmonary blastoma. The so-called pulmonary blastoma of childhood. Cancer. 1988; 62: 1516-26. http://dx.doi.org/10.1002/1097-0142(19881015)62:8<1516::AID-CNCR2820620812>3.0.CO;2-3

[4] Van Loo S, Boeykens E, Stappaerts I, et al. Classic biphasic pulmonary blastoma: a case report and review of the literature. Lung Cancer. 2011; 73: 127-32. PMid: 21513998. http://dx.doi.org/10.1016/j.lungcan.2011.03.018

[5] Travis WD, Brambilla E, Muller-Hermelink HK, et al. World Health Organization Classification of Tumours. Pathology and Genetics of Tumours of the Lung, Pleura, Thymus and Heart. Lyon: IARC Press; 2004.

[6] Smyth RJ, Fabre A, Dodd JD, et al. Pulmonary blastoma: a case report and review of the literature. BMC Res Notes. $2014 ;$ 7: 294. PMid: 24885892. http://dx.doi.org/10.1186/1756-0500-7-294 